\title{
M étodos físicos utilizados para oclusão de varizes dos membros inferiores
}

\author{
Physical methods used to promote ocdusion of varicose veins of the lower limbs
}

\author{
Marcelo Araújoํㅜ Fermin de C. G arcia Velasco ${ }^{2}$
}

\begin{abstract}
Resumo
A terapia das varizes dos membros inferiores tem sido realizada classicamente por cirurgia e escleroterapia, sendo a escolha basicamente dependente do seu cal ibre. Entretanto, a associação de técnicas costuma ser uma necessidade para a obtenção de bons resultados. $0 \mathrm{~s}$ meios físicos surgiram no final da década de 50 e continuaram a progredir com grande diversidade quanto à natureza, princípio físico e efeitos. A complexidade tecnológica é bastante variável. Eletrocoagulação, laser, luz intensa pulsada, crioesclerose endovascular, ultra-som e microondas são meios físicos potencial mente viáveis para esta condição. Entretanto, com algumas exceções, pouco tem sido descrito fora dos centros de pesquisa, e a participação como opção terapêutica ainda necessita de uma melhor definição do papel. 0 artigo tem como objetivo descrever os métodos físicos empregados ou em estudo para a terapia de varizes.
\end{abstract}

Palavras-chave: Varizes, laser, ultra-som, microondas, eletrocoagulação, radiofreqüência, terapia, telangiectasias.

\section{Contexto}

Varizes dos membros inferiores constituem uma condição patológica conhecida há muito tempo, e a busca por uma terapia adequada é tão antiga quanto a própria história da medicina ocidental. Embora a esclerose química seja a forma terapêutica mais difundida e conhecida, a utilização de meios físicos com este fim precedeu-a historicamente. A raspagem e traumatização da veia (efeito mecânico) e a cauterização (efeito

\footnotetext{
1. Pesquisador e professor assistente, Doutor, Departamento de Saúde, Núcleo de Física Médica (FIMED), Universidade Estadual de Santa Cruz, llhéus, BA.

2. Professor Doutor e pesquisador-chefe, FIMED, Universidade Estadual
} de Santa Cruz, Ilhéus, BA.

Artigo submetido em 19.02.06, aceito em 04.05.06.

\begin{abstract}
The therapy of varicose veins of the lower limbs has classically been carried out with surgery and sclerotherapy, depending on vessel diameter. H owever, the association of techniquesis frequently necessary to assure a good result. The use of physical procedures to promote occlusion of varicose veins was firstly attempted in the 1950s. There are different physical principles and effects based on different technological levels. Electrocoagulation, laser, intense pulsed light, endovascular cryosclerosis, ultrasound and microwave are physical sources potentially useful for this condition. Unfortunately, these technologies are not wide available outside research centers, with a few exceptions, and their role in clinical practice still needs to be defined. This paper aims to describe the physical procedurescurrently used or potentially useful for the therapy of varicose veins.
\end{abstract}

Key words: Varicose veins, laser, ultrasound, microwave, electrocoagulation, radiofrequency, therapy, telangiectasias.

térmico), preconizadaspor $\mathrm{H}$ ipócrates ${ }^{1}$ eC elso, respectivamente, são as primeiras referências a este respeito. Em 1667, Elsholtz ${ }^{2}$ utilizou uma técnica rudimentar para a esclerose química de uma variz. $N$ as três situações, o objetivo da destruição da veia foi cicatrizar uma úlcera varicosa. Foi somente com o advento da seringa hipodérmica que, em 1851, Pravaz $^{3}$ descreveu tecnicamente o primeiro processo de esclerose química, neste caso para promover a obliteração de um aneurisma. A partir daí, o processo de escleroterapia química passou a ser utilizado no tratamento das varizes. M elhorias técnicas e de materiais foram introduzidas, contribuindo para uma maior eficiência e conforto da técnica.

Embora bem tolerada, esta técnica é cruenta e dependente da experiência de cada profissional ${ }^{4}$. 0 surgimento de pequenas equimoses, manchas transitórias na pele, desconforto local eraramentesistêmi- 
co pode ocorrer com freqüência variável em função desses fatores. Existem, porém, relatos de complicações indesejáveis, como formação de lesões dérmicas bolhosas e até úlceras cutâneas. A escleroterapia química écertamente a técnica mais difundida e com a maior experiência mundial para o tratamento das telangiectasias. 0 baixo custo do procedimento permitiu a difusão desta modalidade terapêutica, com ampla aceitação por parte dos pacientes e dos médicos. A cirurgia tem sido reservada para veias de maior calibre 4 .

0 interesse por formas alternativas de tratamento de varizes iniciou na década de $50^{5}$. M eios físicos, aparentemente mais sofisticados, menos cruentos e com grande apelo de modernidade, passaram a ser descritos. Atéhoje, novosmeiosfísicos para tratamento de varizes ${ }^{6}$ são descritos com taxas de sucesso variáveis e, em al guns casos, questionáveis.

Todososmétodosfísicostêm na produção do calor ou na ausência dele (o frio), aplicado diretamente ao sangueeem especial ao endotélio vascular, um mecanismo comum na gênese da oclusão vascular. Entretanto, al guns combinam tal aspecto com outras propriedades inerentes ao princípio físico determinante da lesão, como a própria luz e 0 som, por exemplo ${ }^{7}$. 0 papel dessas propriedades será comentado oportunamente. O s níveis de tecnologias empregadas nos meios físicos apresentam um amplo espectro decomplexidade. Classificá-los é tarefa difícil, pelo fato de possuírem ações sinérgicasesimultâneasquenão são mutuamenteexcludentes. Podemos, entretanto, tabular os meios físicos nas categorias abaixo (T abela 1 ).

T abela 1 - T abulação dos meios físicos utilizados no tratamento das varizes dos membros inferiores

\begin{tabular}{|c|c|c|c|c|}
\hline Princípio & M odo/Propriedade & N atureza da ação & Aplicação & Efeitos \\
\hline Eletricidade & Resistividade & $\begin{array}{c}\text { Térmica } \\
\text { (aquecimento) }\end{array}$ & $\begin{array}{l}\text { Percutânea } \\
\text { Endovascular }\end{array}$ & $\begin{array}{l}\text { D iatermo-coagulação } \\
\text { Lesão direta }\end{array}$ \\
\hline Físico-químico & T emperatura & $\begin{array}{c}\text { Térmica } \\
\text { (resfriamento) }\end{array}$ & Endovascular & $\begin{array}{l}\text { D iatermo-coagulação } \\
\text { Lesão direta }\end{array}$ \\
\hline \multirow[t]{4}{*}{$\begin{array}{l}\text { Radiação } \\
\text { eletromagnética }\end{array}$} & Luz - Laser & $\begin{array}{l}\text { Fototérmica } \\
\text { (aquecimento) } \\
\text { Fotoacústica }\end{array}$ & $\begin{array}{l}\text { Percutânea } \\
\text { Endovascular }\end{array}$ & $\begin{array}{c}\text { D iatermo-coagulação } \\
\text { Lesão direta }\end{array}$ \\
\hline & $\begin{array}{l}\text { Luz - Luz policromática } \\
\text { e luz intensa pulsada }\end{array}$ & $\begin{array}{l}\text { Fototérmica } \\
\text { (aquecimento) }\end{array}$ & Percutânea & $\begin{array}{l}\text { D iatermo-coagulação } \\
\text { Lesão direta }\end{array}$ \\
\hline & Radiofreqüência & $\begin{array}{c}\text { Térmica } \\
\text { (aquecimento) }\end{array}$ & Endovascular & $\begin{array}{c}\text { D iatermo-coagulação } \\
\text { Lesão direta }\end{array}$ \\
\hline & M icroondas & $\begin{array}{c}\text { Térmica } \\
\text { (aquecimento) }\end{array}$ & Percutânea & $\begin{array}{l}\text { D iatermo-coagulação } \\
\text { Lesão direta }\end{array}$ \\
\hline \multirow[t]{2}{*}{ Energia acústica } & U Itra-som & $\begin{array}{l}\text { Termo-acústico } \\
\text { (aquecimento) }\end{array}$ & Percutânea & $\begin{array}{l}\text { Cavitação D iater- } \\
\text { mo-coagulação } \\
\text { Ativação plaquetária } \\
\text { Lesão direta }\end{array}$ \\
\hline & Ondas de choque & $\begin{array}{l}\text { Termo-acústico } \\
\text { (aquecimento) }\end{array}$ & Percutânea & $\begin{array}{c}\text { Cavitação D iater- } \\
\text { mo-coagulação } \\
\text { Ativação plaquetária } \\
\text { Lesão direta }\end{array}$ \\
\hline
\end{tabular}


O objetivo deste artigo é revisar e descrever os métodos físicosque têm sido empregados ou propostos para o tratamento das varizes e telangiectasias dos membros inferiores.

\section{M étodos que utilizam predominantemente o princí- pio térmico}

\section{Eletrocoagulação percutânea}

Empregada em veias de menor calibre e, especialmente, nas telangiectasias, a eletrocoagulação foi 0 primeiro método a tornar-se disponível ${ }^{8,9}$. T rata-se de um sistemadebisturi elétrico com freqüênciade 500.000 até $4.000 .000 \mathrm{~Hz}$. A corrente elétrica doméstica de baixa freqüência é transformada em alta freqüência e baixa voltagem, reduzindo-se assim a possibilidade de dano tecidual epromovendo a coagulação das proteínas pelo aumento da temperatura criada no âmago do tecido. N esse caso, o sangue funciona como condutor da energia quegera a el evação da temperatura através do contato direto da ponta unipolar do instrumento. 0 termo eletrofulguração é mais adequado aos casos em que não há um contato direto, mas sim uma proximidadeda ponta unipolar com o tecido, fazendo com que uma centelha atinja o tecido vivo. 0 pção de baixo custo, sua utilização não chegou a ser muito difundida, devido ao desconforto local e à freqüência elevada de lesões cutâneas, ainda quetemporárias. 0 procedimento é doloroso, necessitando freqüentemente anestesia tópica, pois provoca coagulação dos vasos e também queimaduras cutâneas ao redor da agulha, que setransformam em crostas que perduram por 2 a 3 semanas ${ }^{4,10}$. Cicatrizes hipopigmentadas também podem ocorrer, mas a produção de resultados pouco eficientes certamente foi o que mais colaborou para a parca difusão do método. $\mathrm{N}$ aúltima década, foi descrita uma variação técnica procedendo a eletrocoagulação por punção percutânea com el etrodo monoativo; entretanto, também ocorreram queimaduras em al guns casos.

\section{Eletrocoagulação endovascular}

A eletrocoagulação endovascular foi proposta por alguns com a intenção de promover a obliteração de vasos calibrosos, como os troncos safenos ${ }^{5,11-14}$, utilizando o calor. Essa foi a primeira modalidade de tratamento das veiassafenaspor meiosfísicoseminimamente invasivos, sendo um procedimento semelhante ao que se faz hoje com laser e radiofreqüência. 0 método consistia em introduzir um eletrodo ativo na veia safena dissecada distalmente, empregando anestesia e promovendo a termólise endovascular por ação direta. Embora descrita por entusiastas como degrande valor terapêutico e baixo custo, o domínio da técnica ficou restrito a poucos centros.

\section{Crioesclerose endovascular}

D e maneira similar à el etrocoagulação, a crioesclerose por sonda endovascular foi desenvolvida ${ }^{15}$. 0 procedimento também envolve a dissecção da veia safena e introdução de uma sonda, que, nesse caso, produz uma baixa temperatura utilizando-se a rápida expansão de $\mathrm{NO}_{2}$ ou $\mathrm{CO}_{2}$ (efeito J oule-T homson ou J oule-K elvin), com conseqüente queimadura por frio do endotélio. $\mathrm{H}$ abitual mente, real iza-se o congel amento em 10 segundos a cada $2 \mathrm{~cm}$ da veia, recuando-se progressivamentea sonda. A veia obliterada permanece no local. 0 processo inflamatório seguido após isso já foi extensamentedescrito, destacando as peculiaridades da lesão celular induzida pelo frio ${ }^{16}$.

\section{Criocirurgia endovascular}

$\mathrm{N}$ ão se deve confundir com a crioesclerose por sonda endovascular. A técnica é também conhecida como crioinvaginação ou cryostripping e consiste na introdução de uma sonda que, quando posicionada na safena distal, é resfriada, levando ao congelamento em pouco segundos da veia safena ao redor da sonda ${ }^{16}$. O corre então uma forte adesão, possibilitando que a sonda possa ser tracionada à semelhança de um fleboextrator $^{17,18}$. 0 coto distal não precisa ser ligado, poisfica totalmenteobliterado pela ação do frio. $N$ ão hánecessidade de amarrar a veia à sonda, como na fleboextração clássica, e isso é particularmente útil nos casos onde há hipodermite, úlcera ou outras al terações tróficas distais.

\section{Crioescleroterapia percutânea}

A rigor, é uma forma verdadeira de escleroterapia, portanto, será brevemente comentada. A crioescleroterapia consiste em um misto de técnica química e física. A adição do frio potencializa a ação da droga no endotélio vascular ${ }^{19}$. A temperatura do líquido na seringa situa-seem torno de- $40 \stackrel{\circ}{\circ}$, sendo maior ao sair através da agulha ${ }^{20}$. T al resfriamento pode ser obtido por meio de equipamentos refrigeradores especiais ou $\mathrm{CO}_{2}$. Embora utilize punção para injeção endovascular, é considerada uma técnica percutânea, pois não emprega a dissecção cirúrgica clássica. 


\section{M étodos que utilizam o princípio das radiações ele- tromagnéticas}

\section{Laser percutâneo}

0 emprego da técnica de amplificação da luz pela emissão estimulada de radiação, mais conhecida como laser (light amplification by stimulated emission of radiation), surgiu como um método moderno e pouco agressivo, com um grande apelo tecnológico. D esenvolvido a partir da fundamentação teórica concebida por Albert Einstein $(1917)^{21}$, o laser consiste na emissão de um feixe de fótons monocromáticos coerentes e colimados com um comprimento de onda específico. 0 princípio técnico é o resultado da estimulação de um átomo ou molécula, com conseqüente liberação de fótons com comprimento de onda espećífico, que determina, em termos teóricos, a lesão seletiva do vaso preservando a pele. U ma vez que os comprimentos de onda utilizados pelos equipamentos de laser na área médica estão no nível ou próximos à variação da luz visível no espectro eletromagnético, não há, portanto, o risco das radiações ionizantes. A seletividadedo dano depende da absorção pelos cromóforos cutâneos, que constituem uma interfacenaabsorção do feixedeluz. A maioria dosemissores delaser trabal hanafaixa entre $500 \mathrm{e} 600 \mathrm{~nm}$, pois neste espectro observa-se uma maior diferençano coeficiente de absorção entre a melanina e a oxiemoglobina e desoxiemoglobina, o quefacilita a ação sel etiva do laser.

A primeira publicação sobreautilização parafinsde tratamento dastelangiectasias com um laser de $\mathrm{CO}_{2}$ foi feita em 197522. D iferentestipos delaser foram utilizados desde então, com o objetivo de tratar as vênulas e telangiectasias dos membros inferiores e da face, além de outras doenças vasculares cutâneas. 0 dióxido de carbono, N eodímio:YAG, argônio, corante e corante pulsado foram alguns dos mais utilizados nos estudos clínicos. Atualmente, a preferência para tratamento das telangiectasias recai sobre o Frequency D oubled QSwitched N d:YAG (FDQSN d:YAG), um sistema de emissão destravada (que pode ser por prisma giratório, elétrico-óptico ou acústico-óptico por ultra-som) com comprimento de onda de $1.064 \mathrm{~nm}$, que, com a duplicação da freqüência, faz com que o comprimento de onda atinja $532 \mathrm{~nm}$, ficando, portanto, na faixa de absorção adequada conforme mencionado. Esses lasers deemissão destravada ou desen cadeada têm a vantagem de ter uma duração do pulso muito mais curta do que o tempo derelaxamento da pele $\mathrm{e}^{23}$. Como o aquecimento cutâneo costuma causar desconforto, a associação a um dispositivo especial permiteo resfriamento superfi- cial imediato da pele, sendo mais agradável ao paciente. Por outro lado, um resfriamento tecidual profundo, em princípio, pode aumentar a profundidade de absorção, mas acarreta riscos de danos teciduais não-seletivos devido à maior demanda de energia para provocar 0 efeito ${ }^{24}$. 0 desenvolvimento corrente dos emissores de laser já permite a utilização em diferentes tipos de pele e diferentes localizações. A pesar do grande entusiasmo dealgunsmaisotimistas, ostipos $\mathrm{V}$ eVI daclassificação proposta por Fitzpatrick ${ }^{25}$ e os tipos II, III e IV precedentes, quando bronzeados, ainda contra-indicam 0 uso dessa tecnologia pelo risco de maus resultados. Em outras palavras, existe, ainda queem menor escala, uma restrição relativa aos tipos de pele escura ricas em melanina, comuns em países tropicais como o Brasil. Por fim, uma outra limitação da utilização do laser, especialmente em nosso meio, ainda é o seu elevado custo operacional. Conseqüentemente, isso tem retardado a aquisição de experiência com a técnica.

\section{Laser endovascular}

A tendência em realizar procedimentos minimamente invasivos tem feito com que algumas idéias relativamente antigastentem ser melhoradas. A oclusão das veias safenas por métodos diatérmicos tem sido relatada desde os anos 70, através de sondas térmicas introduzidas por dissecção e posicionadas na crossa da safena para obliteração do trajeto venoso varicoso ou incompetente. A idéia é reduzir os riscos e tornar os procedimentosmenoscomplexos, possibilitando intervenções ambulatoriais. D essa forma, reduzir-seia a morbidade e facilitar-se-ia a reintegração mais precoce às atividades. As sondas termogênicas quentes ou frias foram as precursoras. Atualmente, os sistemas de oclusão térmica endovenosa das veias safenas por laser 26-28 já estão sob investigação clínica.

\section{Luz policromática}

Um ano após a publicação do primeiro trabalho sobre a terapia de varizes com laser, experimentou-se um outro processo físico baseado na emissão de luz policromática infravermelha ${ }^{29}$. Esse método não apresentou grande desenvolvimento.

Anos após, o curso da tecnol ogia da luz policromática foi retomado, criando-se então a fonte de luz intensa pul sada ${ }^{30}$, que difere do laser do ponto de vista físico, dentre outros aspectos, pela ausência de um comprimento de onda específico. Atualmente, alguns 
profissionais ainda utilizam esse equipamento, mas o mesmo não oferece vantagens em relação ao laser com comprimento de onda de $1.064 \mathrm{~nm}$. Além disso, esse método mostrou-se menos eficiente no tratamento das telangiectasias. Existem as mesmas limitações do laser quanto ao tipo de pele.

\section{Radiofreqüência}

0 espectro eletromagnético é composto por radiações com faixas de freqüência e, conseqüentemente, comprimento de onda diferente. 0 ndas de rádio, microondas, infravermelho, passando pelo espectro visível, raios ultravioletas, raios $X$ e raios gama compõem este espectro do maior para o menor comprimento de onda progressivamente. Q uanto maior a freqüência, menor o comprimento deonda emaior a capacidade de gerar bioefeitos em condições naturais. As ondas de rádio, utilizadas na radiocomunicação, são as de maior tamanho, variando entre $3 \mathrm{kH}$ ze $300 \mathrm{GH}$ z defreqüência. $N$ essa condição, a radiofreqüência não costuma apresentar efeitos biológicos, mas, quando concentrada e aplicada a áreas restritas, produz ablação tecidual termogênica. Empregada em terapia de tumores (por exemplo, mamários, prostáticos e hepáticos), presta-se também à oclusão das veias tronculares, como as safenas 31,32 . J untamente com o laser, tem sido atualmente proposta como co-intervenção, a fim de reduzir as dificuldades potenciais da abordagem cirúrgica da crossa da safena. 0 mecanismo que promove a oclusão é térmico.

\section{Microondas}

$\mathrm{N}$ o início desta década, foi descrita maisuma opção deterapia para pequenos vasos: a esclerose detelangiectasias por meio da aplicação externa localizada de microondas $^{33}$. M icroondas são ondas do espectro eletromagnético com comprimento de onda menor que a radiofreqüência. $A$ freqüência está situada entre 500 M H z e 300 G H z. O s efeitos são baseados também na produção de calor.

\section{M étodos que utilizam o princípio da energia acústica}

\section{Ultra-som}

UItra-sons são vibrações mecânicas da matéria com freqüência superior a $20.000 \mathrm{~Hz}$ z, limite fisiológico do ouvido humano. A capacidade de o ouvido humano perceber os sons inclui uma faixa espectral que varia de
$16 \mathrm{H}$ z até $20.000 \mathrm{H}$ z. A baixo do limite inferior, considera-se infra-som, e acima do limite superior, ultrasom ${ }^{34}$. A produção do ultra-som pode ser feita por dois processos: magnetostricção e piezoeletricidade. Esta éa mais comum e atinge freqüências mais altas. Q uando a potênciaémaisel evada, costuma-sechamar deondasde choque, como ocorre na litotripsia ultra-sônica, por exemplo.

É muito importante ressaltar que o ultra-som chamado terapêutico utiliza potênciasem torno del a 3 W/ $\mathrm{cm}^{2}$, produzindo efeitos térmicosenão-térmicos. $D$ entre estes, a cavitação e efeitos mecânicos como a micromassagem e o torque acústico são os principais envolvidos na interação tecidual. Já o ultra-som diagnóstico, queconstitui aultra-sonografia, normal menteemprega potências abaixo de $100 \mathrm{~mW} / \mathrm{cm}^{2}$, sendo desprovido de efeitos biológicos sob uso clínico judicioso.

A utilização do ultra-som terapêutico por muito tempo restringiu-se praticamente à fisioterapia e reabilitação física nos traumatismos, doenças do sistema locomotor ${ }^{35}$ e em procedimentos odontológicos ${ }^{36}$. 0 utras aplicações descritas são ressecções prostáticas ${ }^{37}$, facoemulsificação ${ }^{38,39}$, neurocirurgia, cirurgia plástica eressecções hepáticas ${ }^{40,41}$. 0 ultra-som terapêutico foi utilizado para tratamento de varizes por Araújo ${ }^{7}$.

\section{Ondas de choque}

O ndas de choque são essencialmente ondas ultrasônicas deal ta potência. Podemoscitar como aplicações dessa modalidade em outros sistemas biológicos: a litotripsia renal, biliar, pancreática e salivar ${ }^{42}$. 0 desenvolvimento do ultra-som focalizado deal ta intensidade (H IFU S) progrediu como uma vertentediferenciadade suas aplicações. Seu emprego no tratamento dedoenças dos sistemas biológicos, incluindo o sistema vascular, data dos anos 6043,44 .

No ano de 1989, foi publicado o primeiro estudo com a intenção primária de tratar as varizes dos membros inferiores ${ }^{45}$.

M ais tarde, outros autores ${ }^{46-49}$ empregaram $\mathrm{HI}$ FU S com diferentesintensidadesem estudos vasculares experimentais, que confirmaram os danos às paredes vasculares e/ ou trombose.

\section{D iscussão}

Infelizmente, sabe-se muito pouco ainda sobre a natureza da gênese das varizes, e as opções terapêuticas 
limitam-se à ablação dos vasos afetados. N ão há, ainda, terapia que altere o processo gênico. Assim sendo, a prevenção se faz apenas interferindo com os fatores modificáveis, como controlar o peso, estimular o uso da elastocompressão, evitar a utilização de calçados que interfiram com a biomecânica do pé e panturrilha, 0 ortostatismo prolongado, o uso de roupas apertadas, 0 uso de hormônios, etc.

0 objetivo de eliminar as varizes requer freqüentemente a associação de métodos terapêuticos. N os últimos anos, a tradicional cirurgia aumentou seu campo deação, passando a ser empregada mesmo em varizes de pequeno calibreatravés datécnicademini-incisões. Por sua vez, a escleroterapia química, tradicionalmente empregada para terapia de telangiectasias, também tem sido utilizada atualmente em varizes de maior calibre. U ma variação recente nessa técnica passou a empregar o resfriamento da solução (crioescleroterapia), ou seja, manter a glicose hipertônica a $75 \%$ eà temperatura de até - 40 ㄷ através da utilização de dispositivos adaptadosou especialmente desenvolvidos para isso. A crioescleroterapia associa o efeito físico do frio à ação de substâncias químicas. A parenta ser mais vantajosa por reduzir o número de sessões e o desconforto e acelerar os resultados, quando comparada à escleroterapia convencional. Ainda com a intenção de atingir varizes de maior cal ibre, recentemente, passou-seautilizar a escleroterapia com a técnica da espuma. Até mesmo as veias safenas têm sido esclerosadas por esse processo, que, nesse caso, deve ser executado com auxílio da visualização pela ultra-sonografia ${ }^{50}$.

Em se tratando de métodos físicos, o processo que se propõe a gerar uma oclusão venosa permanente e controlada não deve ser chamado de escleroterapia. Segundo a definição dos descritores da $\mathrm{N}$ ational Library of M edicine, esta, por definição etimológica, é uma denominação específica da oclusão venosa obtida por meio de injeções intravenosas de substâncias químicas. O s dicionários de língua portuguesa atribuem o termo esclerose ao endurecimento tecidual resultante de um processo inflamatório, sem especificar a origem. 0 processo de oclusão, nessas situações, não tem denominação específica, exceto no caso dos meios luminosos, que se chama fototermólise. Por analogia e em obediência aos fenômenos físicos encerrados nesses processos, poderíamos atribuir os termos eletrotermólise e sonotermólise aos processos desencadead os pela el etricidadee pelo som, respectivamente.
A utilização dos meios físicos tem sido feita tanto em telangiectasias como em varizes maiores. $\mathrm{N}$ a grande maioriados casos, constitui um tempo ou uma etapa do tratamento clínico ou cirúrgico. U ma crítica fundamental que se pode fazer aos métodos físicos para oclusão, assim como à esclerose com espuma das veias safenas, é que eles destroem exatamente as veias mais úteis para revascularizações, o oposto do que se propõe com a moderna cirurgia que visa preservá-las ${ }^{51}$. D entre os métodosfísicos citados, somente o laser ea radiofreqüência endovascular, particularmente para oclusão das safenas ${ }^{52}$, e o laser percutâneo, para as telangiectasias, têm sido correntemente usados na clínica. M icroondas ${ }^{33}$ eultra-som percutâneo ${ }^{7}$ estão em investigação experimental. A eletrocoagulação está em desuso ${ }^{4}$. O s demais tiveram ou têm uso restrito e não definiram 0 papel no leque de opções terapêuticas.

O smétodosfísicosainda não ocupam um papel de destaque na terapia das varizes dos membros inferiores. Seja por questões decusto ou por limitações próprias da técnica e da metodologia aplicada, os métodos físicos ainda são restritos a al guns profissionais ou centros de excelência. Embora comercializados e utilizados clinicamente em alguns países, muitas dessas aplicações são experimentais.

$\mathrm{N}$ a medicina, o avanço tecnológico sempre vem acompanhado de uma el evação de custos, por conta da capacitação do profissional para realização do procedimento eda aquisição emanutenção do equipamento. 0 bom uso da tecnologia depende do conhecimento básico da matéria, da avaliação criteriosa dos trabalhos de pesquisa edeum senso ético apurado. Entender as bases dos métodos físicos reveste-se de importância para o médico que se dedica ao estudo e tratamento das doenças do campo da flebologia. N uma época em que as fronteiras de mercado tendem a tornar-se tênues e vulneráveis, essa atitude familiariza e ajuda a assegurar que o profissional médico é real mente capaz e necessário para indicar e realizar ou não o procedimento. A modernização e o avanço tecnológico são inerentes ao desenvolvimento humano, e qualquer ganho de qualidade certamente será incorporado na boa prática da medicina.

\section{Agradecimentos}

À Profa. M Sc. Agnes M aria da Fonseca Fausto, pesquisadora do $\mathrm{N}$ úcleo de Física M édica (FIM ED), U niversidade Estadual de Santa C ruz, IIhéus, BA. 


\section{R eferências}

1. H ippocrates, 400 BC. On ulcers. In: Stevenson DC. The Internet Classics Archive. Disponível em: http:// www.classics.mit.edu/H ippocrates/ulcers.mb.txt. Acessado: 04/03/2004.

2. ElsholtzJS. Clysmatica nova: sive, ratio, qua in venam sectam medicamenta immitti possint, ut eodem modo, ac si per os assumta fuissent, operentur: addita etiam omnibus seculis inaudita sanguinistransfusione. $2^{a}$ ed. Berlin, 1667. D isponível em: http://www.nlm.nih.gov/. A cessado: 04/03/2004.

3. Pravaz J-C-T. Essai sur le traitement des anéurismes par lês injections de perchlorure de fer. Paris, 1857. Disponível em: http://www.nlm.nih.gov/. Acessado: 04/03/2004.

4. Silveira PRM . A escleroterapia hoje. Rev Angiol Cir Vasc. 1993;2:144-7.

5. H ejhal L, Firt P, LivoraD. Endovascular electrocoagulation of superficial varices of leg. Rozhl Chir. 1959;38:418-25.

6. T eruya TH , Ballard JL. N ew approaches for the treatment of varicose veins. Surg Clin N orth Am. 2004;84:1397-417.

7. Araújo M . Terapiaultra-sônica oclusora percutânea das varizes etelangiectasiasdosmembrosinferiores: ensaio clínico al eatório [tese]. São Paulo: U niversidade Federal de São Paulo, Escola Paulista de M edicina; 2004.

8. W erner G, Alexander HA, M CPheeters HO. Electrofulguration: new surgical method for varicose veins. M inn M ed. 1964;47:255-7.

9. Schanno JF. Electrocoagulation: a critical analysis of its useas an adjunct in surgery for varicose veins. Angiology. 1968;19: 288-92.

10. M iyakeRK, M iyakeH, D uarteFH, FidelisRJR. M icrovarizes e telangiectasias. In: Pitta GBB, Castro AA, Burihan E, editores. Angiologia e cirurgia vascular: guia ilustrado [livro on-line]. M aceió: U ncisal/ECM AL; 2003. Disponível em: http://www.lava.med.br/livro.

11. M usaev SM. Intravascular electrocoagulation of dilated subcutaneous varicose veins of the lower extremities. Eksp Khir Anesteziol. 1963;27:36-7.

12. Politowski M, Zelazny T. Complications and difficulties in electrocoagulation of varices of thelower extremities. Surgery. 1966;59:932-4.

13. W atts GT. Endovenous diathermy destruction of internal saphenous. Br M ed J. 1972;4:53.

14. 0 'Reilly K. Letter: endovenousdiathermy sclerosisasa unit of the armamentarium for the attack on varicose veins. $M$ ed J Aust. 1974;1:900.

15. M illeret $R$, Le Pivert $P$. C ryosclerosis of the saphenous veins in varicose reflux in the obese and elderly. Phlebologie. 1981;34:601-5.

16. Le Pivert P. Controlled cryosurgery of varices of the lower extremities. A new therapeutic approach. Apropos of 350 cases. Phlebologie. 1987;40:123-48.

17. Etienne G, Constantin JM, H evia M. Cryo-stripping: an advance in the treatment of varicose veins. 3811 operated limbs. Presse M ed. 1995;24:1017-20.

18. B reuninger $\mathrm{H}$. C ryostripping of the long saphenous vein with a percutaneously guided probe. Dermatol Surg. 2001;27: 545-8.

19. Ripoll-Sanchez M . Presentación de una técnica: crioesclerosis líquida. Rev Soc Esp M ed Estet. 1995;39:19-24.
20. FerreiraM V, Seidel AC, Fregadolli LV, Borghesan CE. Aferição da temperatura da glicose utilizada na crioescleroterapia. J Vasc Bras. 2005;4:155-60.

21. Einstein A. Zur Quantentheorie der Strahlung. Physiol. 1917;18:121-8.

22. K aplan I, Peled I. Thecarbon dioxidelaser in thetreatment of superficial telangiectasias. Br J Plast Surg. 1975;28:214-5.

23. $M$ aillet $H$, coordenador. $O$ laser: princípios e técnicas de aplicação. São Paulo: M anole; 1987.

24. H aina $D$, Landthaler $M$, Braun-Falco $O, W$ aidelich $W$. Comparison of maximum coagulation depth in human skin for different types of medical lasers. Lasers Surg M ed. 1987;7:355-62.

25. Fitzpatrick TB. Soleil et peau. J M ed Esthet. 1975;2:33-7.

26. $N$ avarro L, M in RJ, BoneC. Endovenouslaser: anew minimally invasive method of treatment for varicose veins - preliminary observations using an $810 \mathrm{~nm}$ diode laser. Dermatol Surg. 2001;27:117-22.

27. ProebstleTM, Lehr H A, Kargl A, et al. Endovenoustreatment of the greater saphenous vein with $940 \mathrm{~nm}$ diode laser: thrombotic occlusion after endoluminal thermal damage by laser-generated steam bubbles. J V asc Surg. 2002;35:729-36.

28. Parente EJ, Rosenblatt M. Endovenous laser treatment to promotevenous occlusion. Lasers Surg M ed. 2003;33:115-8.

29. M uhlbauer $W, N$ ath $G, K$ reitmair $A$. T reatment of capillary hemangiomas and nevi flammei with light. Langenbecks Arch Chir. 1976; Suppl:91-4.

30. Goldman MP, Eckhouse S. Photothermal sclerosis of leg veins. ESC M edical Systems, LT D Photoderm V L C ooperative Study G roup. Dermatol Surg. 1996;22:323-30.

31. Goldman M P. Closure of the greater saphenous vein with endoluminal radiofrequency thermal heating of the vein wall in combination with ambulatory phlebectomy: preliminary 6-month follow-up. D ermatol Surg. 2000;26:452-6.

32. W eissRA, W eiss M A. C ontrolled radiofrequency endovenous occlusion usingauniqueradi ofrequency catheter under duplex guidance to eliminate saphenous varicose vein reflux: a 2-year follow-up. D ermatol Surg. 2002;28:38-42.

33. Lowe N J, Grozdev B, M oore D. M icrowave delivery system for lower leg telangiectasias. J C utan Laser Ther. 2000;2:3-7.

34. Grémy $F$, Pagès J-C. Éléments de biophysique. Paris: Flammarion; 1966.

35. D ocker M F, Foulkes D J, Patrick M K. U Itrasound couplants for physiotherapy. Physiotherapy. 1982;68:124-5.

36. Forrest J0. Ultrasonic scaling. A five-year assessment. $\mathrm{Br}$ D ent J. 1967;122:9-14.

37. Bihrle R, Foster RS, Sanghvi NT, Donohue JP, H ood PJ. $\mathrm{H}$ igh intensity focused ultrasound for the treatment of benign prostatic hyperplasia: early U nited States clinical experience. J U rol. 1994;151:1271-5.

38. Kelman CD. Phaco-emulsification and aspiration. A new technique of cataract removal. A preliminary report. Am J O phthalmol. 1967;64:23-35.

39. Kelman CD . Phaco-emulsification and aspiration. A report of 500 consecutive cases. Am J O phthalmol. 1973;75:764-8.

40. Bruno G, Amadei F, Abbiati G . Liposculpturewith ultrasound: biomedical considerations. Aesthetic Plast Surg. 1998;22: 401-3. 
41. W uchinich D. U Itrasonic surgical aspiration: instrumentation, clinical experience and safety. Plast Reconstr Surg. 1998;102:281-3.

42. Belcaro G, Nicolaides AN, M arlinghaus EH, et al. Shock waves in vascular diseases: an in-vitro study. Angiology. 1998;49:777-88.

43. LelePP. Production of deep focal lesionsby focused ultrasound - current status. U Itrasonics. 1967;5:105-12.

44. FryFJ, Kossoff G, Eggleton RC, D unn F. Threshold ultrasonic dosages for structural changes in the mammalian brain. J Acoust Soc Am. 1970;48:1413+.

45. Schultz-Haakh H, Li JK, W elkowitz W, Rosenberg N. U Itrasonic treatment of varicose veins. Angiology. 1989;40:129-37.

46. Yang R, G rififith SL, Rescorla FJ, et al. Feasibility of using high intensity focused ultrasound for the treatment of unresectable retroperitoneal malignancies. In: 36th Annual Convention: Preprinted Abstractsand Programme; 1992; San $D$ iego. Laurel: American Institute of U Itrasound in M edicine; 1992.

47. Seidl M, Steinbach P, H ofstadter F. Shock wave induced endothelial damage - in situ analysis by confocal laser microscopy. Ultrasound M ed Biol. 1994;20:571-8.

48. D elon-M artin C, V ogt C, C hignier E, Guers C, C hapelon JY, $C$ athignol $D$. Venous thrombosis generation by means of high-intensity focused ultrasound. Ultrasound Med Biol. 1995;21:113-9.
49. H ynynen K, Chung AH, Colucci V, Jolesz FA. Potential adverse effects of high-intensity focused ultrasound exposure on blood vessels in vivo. Ultrasound M ed Biol. 1996;22: 193-201.

50. Breu FX, G uggenbichler S. European C onsensus M eeting on Foam Sclerotherapy, April 4-6, 2003, T egernsee, G ermany. D ermatol Surg. 2004;30:709-17.

51. Pitta GBB, C astro AA, T eixeira LR, Francisco J r J, M iranda $J r F$, Burihan $E$. Preservation of the greater saphenous vein in primary troncular varicose vein surgery. J V asc Bras. 2002;1: 32-8.

52. W eissRA. Endovenoustechniquesfor elimination of saphenous reflux: a valuable treatment modality. Dermatol Surg. 2001;27:902-5.

\section{Correspondência:}

M arcelo Araújo

Clínica M ed Angio, Centro M édico Artumiro Fontes

Av. Aziz M aron, 1117/501

CEP 456005-415 - Itabuna, BA

Tel./Fax: (73) 3617.0011

E-mail: marceloaraujo@uesc.br

\section{Aviso aos ex-residentes}

Se você foi residente até 2005 e ainda não está inscrito em sua R egional, procure regularizar sua situação para passar a receber o J Vasc B ras imediatamente, sem qualquer ônus.

Ajude a manter 0 cadastro da SBACV atualizado. 